\title{
Efficiency and Selectivity Aspects in the C-H Functionalization of Aliphatic Oxygen Heterocycles by Photocatalytic Hydrogen Atom Transfer
}

\section{Carlotta Raviola \\ Davide Ravelli* (])}

PhotoGreen Laboratory, Department of Chemistry, University of

Pavia, viale Taramelli 12, 27100 Pavia, Italy

davide.ravelli@unipv.it

Published as part of the Special Section 10th EuCheMS Organic Division Young Investigator Workshop

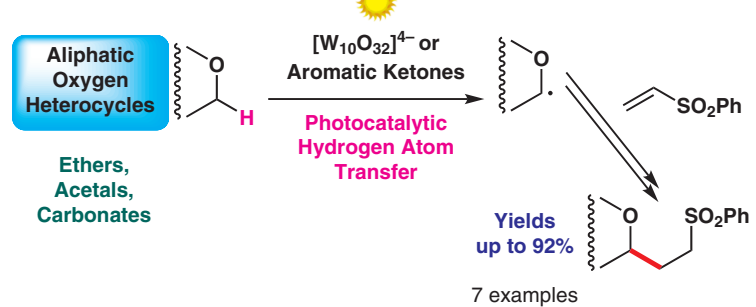

7 examples
Received: 05.12.2018

Accepted after revision: 01.01.2019

Published online: 05.02.2019

DOI: 10.1055/s-0037-1612079; Art ID: st-2018-v0788-I

License terms: cc)

Abstract The $\mathrm{C}-\mathrm{H}$ to $\mathrm{C}-\mathrm{C}$ conversion in aliphatic oxygen heterocycles (dioxolanes, 1,3-dioxane, or cyclic carbonates) by photocatalytic hydrogen atom transfer and subsequent trapping of the resulting radical with phenyl vinyl sulfone was investigated. The performance of three different photocatalysts, namely tetrabutylammonium decatungstate and the aromatic ketones thioxanthone and 9-fluorenone, was compared. The UV-light-absorbing decatungstate anion is more efficient and permits the use of a smaller excess of hydrogen donor than the aromatic ketones, although the ketones could be excited by visible light. Further intramolecular selectivity studies revealed that aromatic ketones afforded a higher proportion of functionalization at the acetalic versus the ethereal positions than did the decatungstate anion.

Key words photocatalysis, hydrogen atom transfer, oxygen heterocycles, chemoselectivity, radicals, C-C bond formation

Oxygen heterocycles are an important family of heterocyclic systems. ${ }^{1}$ These privileged structures are present in many naturally occurring compounds, notably carbohydrates and nucleosides among biomolecules, as well as in a plethora of compounds having pharmaceutical activities. According to a recent report, oxygenated derivatives are the second-most-common type of heterocycles present as a structural motif in pharmaceuticals approved by the US Food and Drug Administration. Interestingly, the majority (around 90\%) of the encountered oxygen heterocycles are nonaromatic in character, with pyranoses, furanoses, macrolactones, morpholines, and dioxolanes occupying the first five positions in the ranking in terms of their relative abundance. ${ }^{2}$ Accordingly, the construction and functionalization of these scaffolds is an important topic for synthetic chemists. A classic example is the Paal-Knorr synthesis of furans,

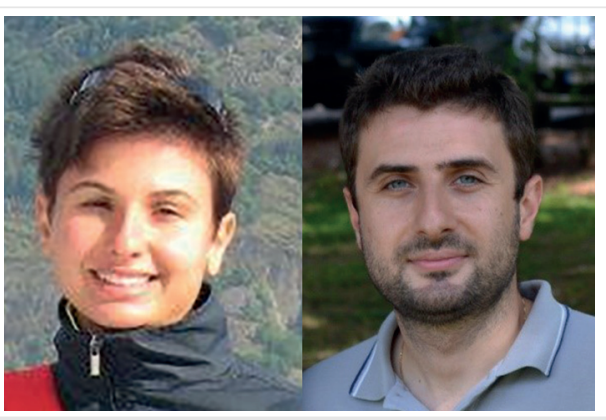

Carlotta Raviola received her Ph.D. from the University of Pavia in 2015 (supervisor: Professor A. Albini), having spent part of her study period at TUM (Germany) in the group of Professor T. Bach. She is currently a postdoctoral researcher at the University of Pavia, and her research interests focus on the photogeneration of highly reactive intermediates (aryl cations, radicals, and diradicals) for use in in organic synthesis.

Davide Ravelli is currently a researcher at the PhotoGreen Laboratory of the University of Pavia. His main research interests are in the area of photochemical reactions and their attending applications in various fields, particularly in organic synthesis. He is very interested in the discovery of methods for the facile generation of valuable intermediates (mainly radicals). In recent years, he has been deeply involved in the study of decatungstate-mediated photocatalyzed reactions (particularly, those involving a hydrogen atom transfer step).

whereas more-recent strategies make extensive use of transition-metal catalysis. ${ }^{3}$ Interestingly, microwave-assisted protocols have also been developed. ${ }^{4}$

An intriguing alternative is represented by photocatalytic strategies, ${ }^{5-7}$ in which a photoactive catalyst is responsible for light absorption and, once in its excited state, for the activation of the actual substrate of the process. ${ }^{8}$ This step leads to the formation of highly reactive intermediates, mostly open-shell radical ions or radicals, which, in turn, are responsible for the observed chemistry. In the particular case of aliphatic oxygen heterocycles, the photocatalytic approach opens the way to the direct functionalization of 
C-H bonds through a hydrogen atom transfer (HAT) step. ${ }^{9}$ Thus, a few photocatalysts ${ }^{10}$ or photogenerated HAT reagents ${ }^{11}$ are capable of directly cleaving $\mathrm{C}-\mathrm{H}$ bonds in the starting material. Along this line, our group recently explored the use of the decatungstate anion, as its tetrabutylammonium salt $\left[\left(\mathrm{Bu}_{4} \mathrm{~N}\right)_{4}\left(\mathrm{~W}_{10} \mathrm{O}_{32}\right)\right.$; TBADT] as a photocatalyst, thanks to the peculiar reactivity of its excited state, which has a partial alkoxy-radical character. ${ }^{12,13}$ Thus, TBADT smoothly promoted $\mathrm{C}-\mathrm{H}$ to $\mathrm{C}-\mathrm{C}$ conversions in various hydrogen donors, including tetrahydrofuran (THF), 1,4dioxane, oxetanes, 1,3-benzodioxoles, and a few lactones, through trapping of the photogenerated radicals with electron-deficient olefins ( $\alpha, \beta$-unsaturated esters, ketones, nitriles, sulfones, etc.) in a conjugate radical addition process [see Scheme 1(a)]. Upon back-HAT, the resulting radical adduct finally led to the functionalized heterocycle, while the photocatalyst was restored to its original state. ${ }^{12,13}$ The reactions were routinely carried out under UV irradiation $\left(\lambda_{\mathrm{EXC}}=310\right.$ or $\left.366 \mathrm{~nm}\right)$, but solar radiation was also demonstrated to be a convenient choice. ${ }^{12}$
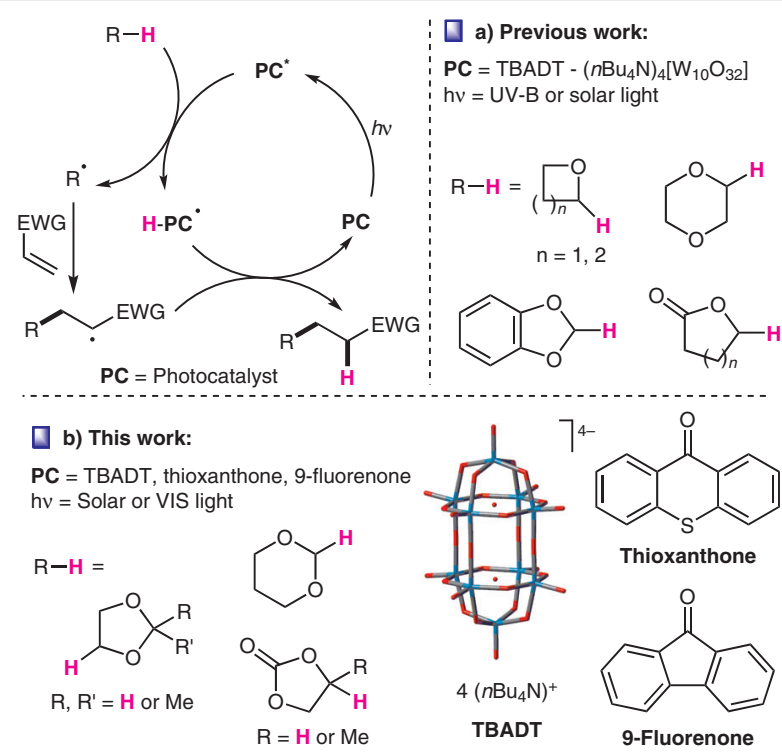

Scheme 1 C-H functionalization of aliphatic oxygen heterocycles through photocatalytic hydrogen atom transfer

Other groups have exploited the similar HAT reactivity displayed by the excited states of aromatic ketones (e.g., benzophenone, 4-benzoylpyridine, quinones, etc. $)^{10 b, 14,15}$ and by the dye eosin $\mathrm{Y}^{16}$ in a wide range of synthetically appealing processes, including $\mathrm{C}-\mathrm{C},{ }^{17} \mathrm{C}-\mathrm{N},{ }^{18}$ and $\mathrm{C}$-halogen ${ }^{19}$ bond-forming reactions, as well as in oxidations, ${ }^{20}$ among others. Indeed, a few of these photoorganocatalysts (POCs) ${ }^{7}$ have also been demonstrated to operate under visible-light irradiation. ${ }^{16,17 \mathrm{~d}-\mathrm{f}, 18-20}$
In this work, we offer a direct comparison of the different reactivities shown by the decatungstate anion and by a selection of (visible-light absorbing) aromatic ketones in the functionalization through photocatalytic HAT of selected families of aliphatic oxygen heterocycles. Furthermore, we also investigated how the choice of the photocatalyst modifies the intramolecular chemoselectivity of the $\mathrm{C}-\mathrm{H}$ cleavage step [Scheme 1(b)]. At the beginning, we performed an optimization of the reaction conditions by studying the addition of THF (1a) to phenyl vinyl sulfone (2), taken as a model trap and then used throughout the rest of the work, to give the adduct $\mathbf{3 a}$ in the presence of selected photocatalysts [Scheme 1(b)]. ${ }^{21}$ We had already investigated this reaction in the presence of TBADT ( $2 \mathrm{~mol} \%$ ), and we obtained $\mathbf{3 a}$ in $59 \%$ yield upon irradiation of a $\mathrm{MeCN}$ solution containing $1 \mathbf{1 a}$ (5 equiv) and $2(0.1 \mathrm{M})$ for 24 hours with phosphor-coated lamps $(12 \times 15 \mathrm{~W})$ that emitted radiation centered at $366 \mathrm{~nm} .{ }^{22}$ Interestingly, the yield of 3a increased to $90 \%$ (as determined by GC analysis) when the irradiation was performed for 20 hours in a solar simulator equipped with a $1500 \mathrm{~W}$ xenon lamp $\left(500 \mathrm{~W} \cdot \mathrm{m}^{-2}\right.$ light intensity; see Table 1 , entry 1 ). When the reaction was performed on a $0.5 \mathrm{mmol}$ scale, 3a was isolated in $86 \%$ yield after silica-gel chromatography. Next, we tested the aromatic ketones thioxanthone and 9-fluorenone as POCs ${ }^{7}$ under analogous reaction conditions. Thus, irradiation of a solution of 1a and $\mathbf{2}$ in the presence of thioxanthone (20 $\mathrm{mol} \%$ ) in dichloromethane (for reasons of solubility) under solar-simulated conditions led to a reasonable consumption of 2 (64\%), with the formation of $\mathbf{3 a}$ in $88 \%$ yield based on the consumed 2 ( $56 \%$ overall yield; entry 2 ). In the presence of 9-fluorenone (20 mol\%), the reaction gave poor results, and 3a was obtained in only $33 \%$ yield at $24 \%$ consumption of 2 (entry 3). Because they can absorb in the visible-light range [see Supporting Information (SI); Figure S1], aromatic ketones can also operate as POCs upon irradiation at $\lambda>400$ $\mathrm{nm}$. We therefore tested their reactivities upon irradiation with a $1 \mathrm{~W}$ violet $\operatorname{LED}\left(\lambda=405 \pm 5 \mathrm{~nm} ; 130 \mathrm{~W} \cdot \mathrm{m}^{-2}\right.$ light intensity). With both POCs, product 3a was formed in a low yield and, again, with incomplete consumption of $\mathbf{2}$ (entries 4 and 5). In the attempt to improve both the yield and the conversion of the starting olefin, we decided to adopt a greater excess of the hydrogen donor ( $3 \mathrm{M}$; 30 equiv). Interestingly, in the case of thioxanthone, the desired adduct 3a was obtained in $87 \%$ isolated yield (entry 6 ), a similar value to what obtained with TBADT (entry 1). On the other hand, with 9-fluorenone, we failed to achieve complete consumption of $\mathbf{2}$, even after irradiation for 30 hours (entries 7 and 8). Importantly, in all cases, the reaction required both light and the presence of the chosen photocatalyst to proceed, as demonstrated by blank experiments (See SI; Table S1). With these preliminary results in hand, we extended the reaction to 2,2-dimethyl-1,3-dioxolane (1) $\mathbf{b})$. The reaction proceeded satisfactorily in the presence of TBADT, giving $\mathbf{3 b}$ in $55 \%$ iso- 
lated yield with a complete consumption of $\mathbf{2}$ (entry 9). On the other hand, aromatic ketones gave unsatisfactory results, even when $\mathbf{1 b}$ was used in a large excess (50 equiv), and the yields were around 10\% (entries 10 and 11). Finally, we tested the reactivity of a cyclic carbonate, namely ethylene carbonate (1c), as a hydrogen donor. In this case, the efficiency of the process dropped significantly, and $\mathbf{3 c}$ was obtained in only $57 \%$ isolated yield in the presence of TBADT and 50 equiv of 1c (entries 12-14), whereas both the investigated POCs failed to give the desired adduct $\mathbf{3 c}$ (entries 15 and 16).

Table 1 Optimization of the Reaction Conditions, and Initial Experiments $^{\mathrm{a}}$

\begin{tabular}{|c|c|c|c|}
\hline Entry 1 (M) & Conditions $^{\mathrm{b}}$ & $\begin{array}{l}\text { Consumption }{ }^{c} \\
\text { (\%) of } \mathbf{2}\end{array}$ & $\begin{array}{l}\text { Yieldc } \\
\text { (\%) of } 3\end{array}$ \\
\hline
\end{tabular}

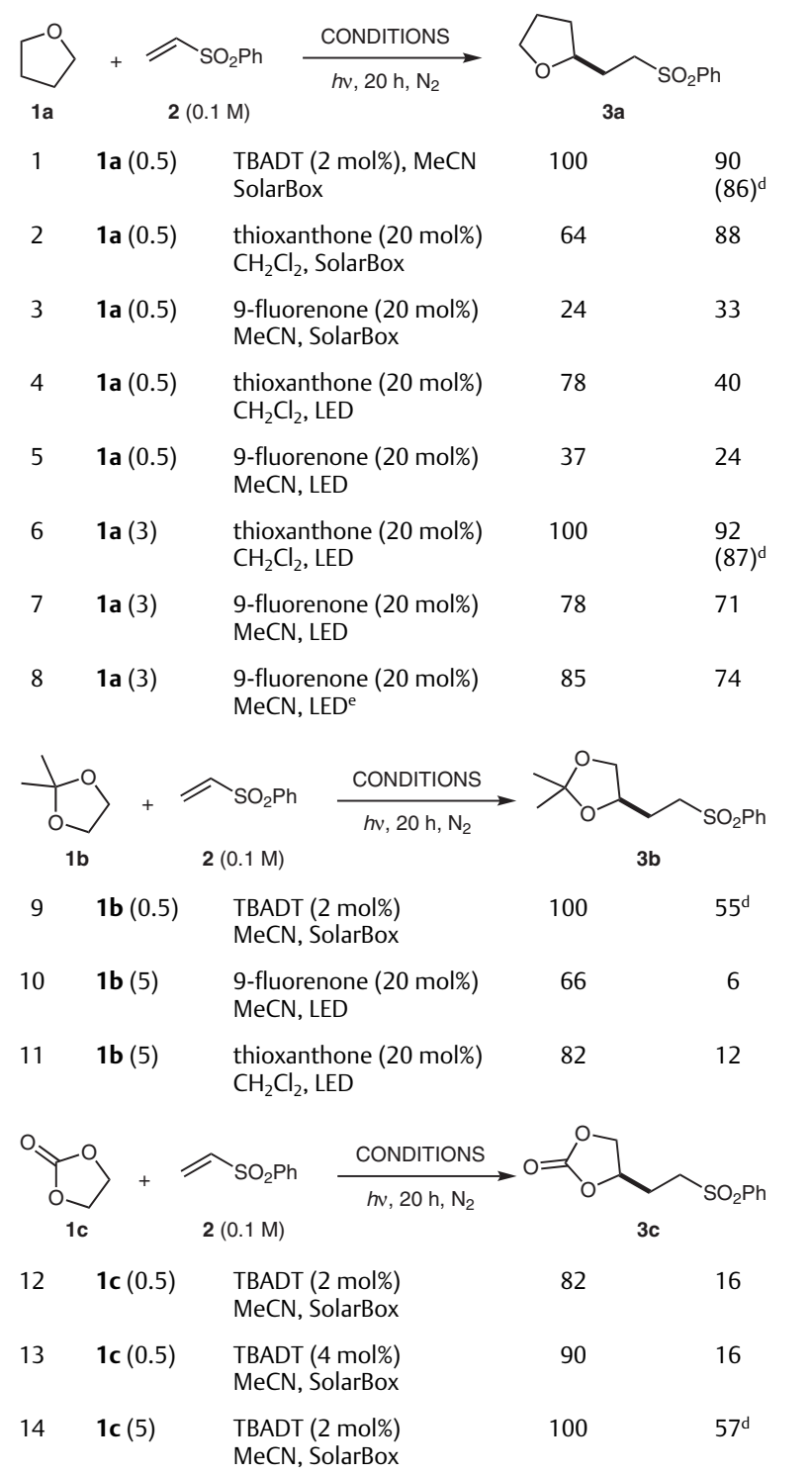

\begin{tabular}{|c|c|c|c|c|}
\hline Entry & $1(\mathrm{M})$ & Conditions $^{\mathrm{b}}$ & $\begin{array}{l}\text { Consumption }{ }^{c} \\
\text { (\%) of } \mathbf{2}\end{array}$ & $\begin{array}{l}\text { Yieldc } \\
\text { (\%) of } 3\end{array}$ \\
\hline 15 & $\mathbf{1 c}(5)$ & $\begin{array}{l}\text { 9-fluorenone (20 mol\%) } \\
\text { MeCN, LED }\end{array}$ & 21 & n.d. ${ }^{f}$ \\
\hline 16 & 1c (5) & $\begin{array}{l}\text { thioxanthone (20 mol\%) } \\
\mathrm{CH}_{2} \mathrm{Cl}_{2} \text {, LED }\end{array}$ & 40 & n.d. \\
\hline
\end{tabular}

a Irradiations were carried out in Pyrex vials on a $0.1 \mathrm{mmol}$ scale with $\mathrm{MeCN}$ (for TBADT or 9-fluorenone) or $\mathrm{CH}_{2} \mathrm{Cl}_{2}$ (for thioxanthone) as the solvent. Solutions were bubbled with $\mathrm{N}_{2}$ for 5 min before irradiation.

${ }^{b}$ SolarBox: irradiation was performed with a solar-light simulator equipped with a $1.5 \mathrm{~kW}$ Xe lamp $\left(500 \mathrm{~W} \cdot \mathrm{m}^{-2}\right.$ light intensity). LED: irradiation was per-

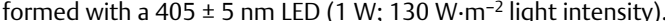

' Except where otherwise noted, the consumption of $\mathbf{2}$ and the yield of $\mathbf{3}$ were determined by GC with dodecane $\left(1 \mu \mathrm{L} \cdot \mathrm{mL}^{-1}\right)$ as internal standard. Yields of $\mathbf{3}$ are expressed on the basis of the consumed $\mathbf{2}$ and can interpreted as 'selectivity' values.

d Isolated yield from a reaction performed on a $0.5 \mathrm{mmol}$ scale after chromatography (silica gel, cyclohexane-EtOAc).

e Irradiation for $30 \mathrm{~h}$.

${ }^{\mathrm{f}}$ n.d. $=$ not detected

To examine the possibility of performing selective $\mathrm{C}-\mathrm{H}$ to $\mathrm{C}-\mathrm{C}$ conversions, we then moved on to study a series of hydrogen donors containing various positions prone to functionalization, and the results are summarized in Table 2. We initially tested the reactivity of 1,3-dioxolane (1d) in the presence of the same photocatalysts as used above under conditions similar to those adopted for $\mathbf{1 a}$ and $\mathbf{1 b}$. Thus, the use of TBADT gave a $42 \%$ overall yield of a $58: 42$ mixture of products $\mathbf{3 d}$ and $\mathbf{4 d}$, resulting from the functionalization at the acetalic and ethereal positions, respectively (Table 2 , entry 1 ).

Table 2 Intramolecular selectivities in the $\mathrm{C}-\mathrm{H}$ to $\mathrm{C}-\mathrm{C}$ functionalization of aliphatic oxygen heterocycles. ${ }^{\text {a }}$

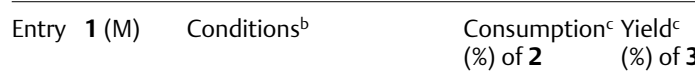

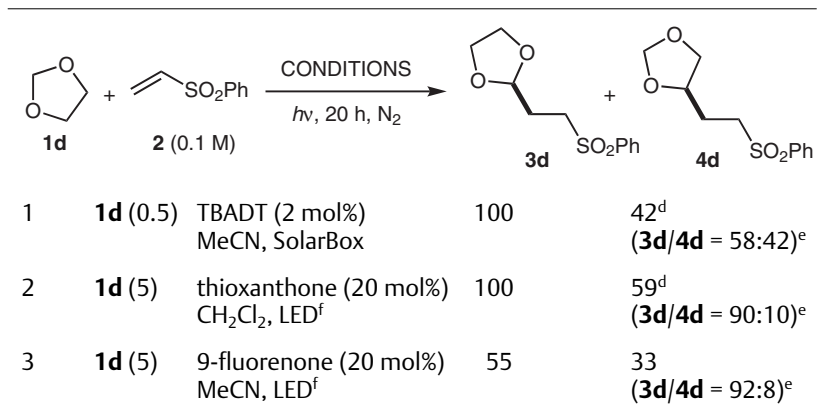

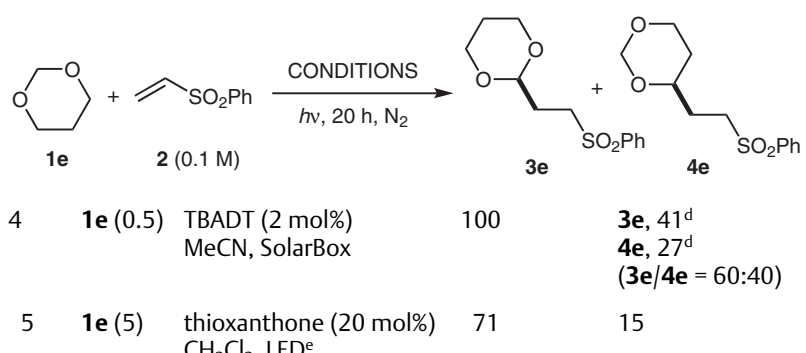


Table 2 (continued)

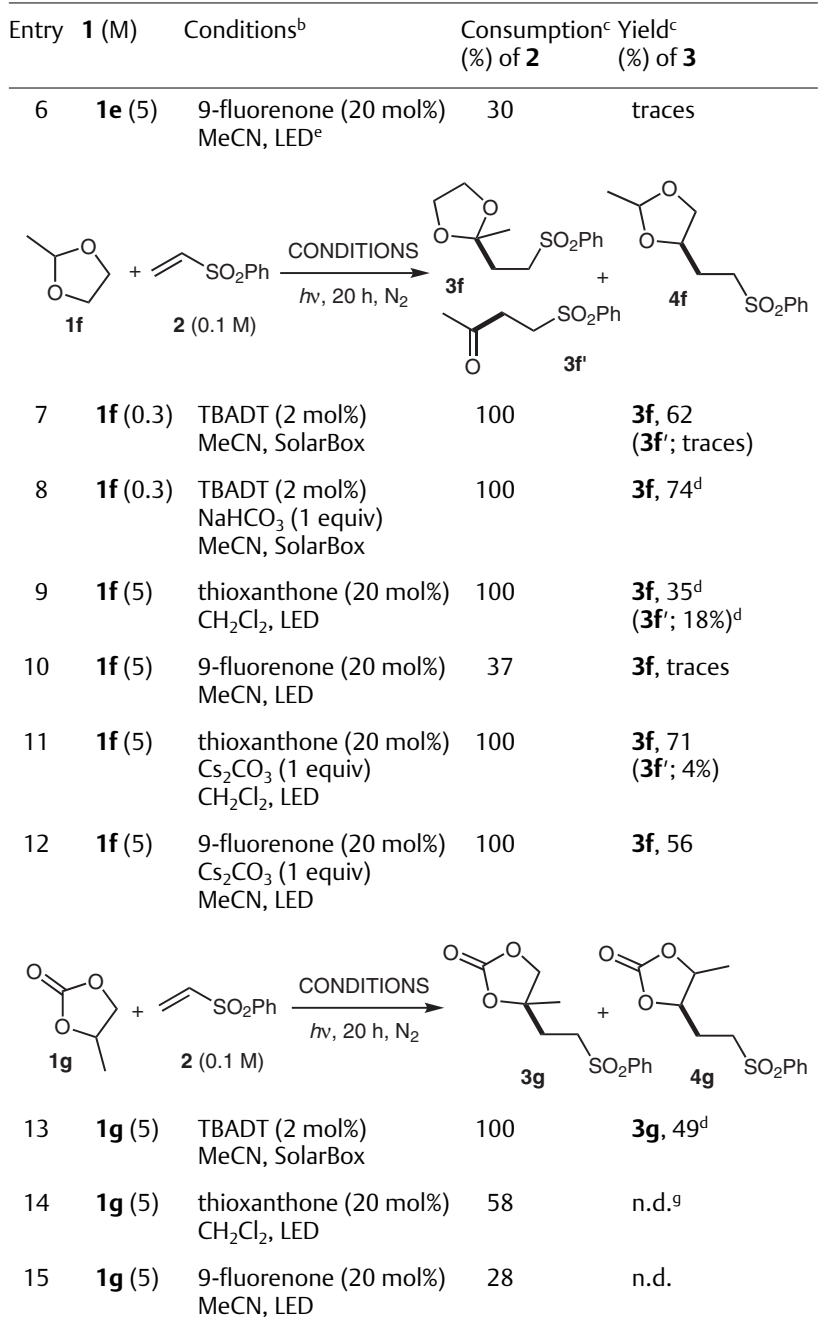

a-d See Table 1.

e The ratio of $\mathbf{3}$ to $\mathbf{4}$ was determined by ${ }^{1} \mathrm{H}$ NMR (see SI).

f Irradiation for $30 \mathrm{~h}$.

${ }^{g}$ n.d. $=$ not detected

Whereas the hydrogen donor was used in a fivefold excess in the case of TBADT, thioxanthone required the use of 50 equivalents of $\mathbf{1 d}$, affording a 90:10 mixture of $\mathbf{3 d}$ and 4d in 59\% overall yield upon irradiation for $30 \mathrm{~h}$ (Table 2, entry 2). A similar product ratio was consistently obtained when 9-fluorenone was used as the POC, although the desired adduct was formed in low yield and with a partial consumption of $\mathbf{2}$ (entry 3 ). Next, we moved to six-membered oxygen heterocycles, and we used 1,3-dioxane (1e) as the hydrogen donor. The reaction in the presence of TBADT afforded two different products, 3 e (41\% isolated yield) and 4e (27\% isolated yield), resulting from the functionalization at the acetalic and ethereal positions, respectively $(\mathbf{3 e} / \mathbf{4 e}=$ 60:40; entry 4). On the other hand, thioxanthone promoted the exclusive formation of $\mathbf{3 e}$, albeit with a poor perfor- mance according to GC analysis, whereas 9-fluorenone only gave traces of the desired adduct $3 e$ (entries 5 and 6). On changing to 2-methyl-1,3-dioxolane (1f), which has a tertiary acetalic position, the reaction in the presence of TBADT gave full consumption of $\mathbf{2}$ in the presence of only a threefold excess of 1f. Indeed, the functionalization at the acetalic position occurred exclusively, although the desired product $3 \mathbf{f}$ was accompanied by traces of compound 3f' resulting from deprotection of the acetal group. Notably, the formation of this undesired byproduct could be suppressed by performing the reaction in the presence of a mild base $\left(\mathrm{NaHCO}_{3}, 1\right.$ equiv $),{ }^{23}$ giving product $\mathbf{3 f}$ as the only isolated adduct in $74 \%$ yield (entries 7 and 8). Thioxanthone or 9fluorenone again required the use of a large excess of hydrogen donor (50 equiv). In the first case, $\mathbf{3 f}$ was formed in $35 \%$ yield along with $18 \%$ of $\mathbf{3 f}^{\prime}$, whereas in the latter case, only traces of the adduct $3 \mathbf{f}$ were found by GC analysis (entries 9 and 10). In both cases, the addition of $\mathrm{Cs}_{2} \mathrm{CO}_{3}$ (1 equiv) pushed the reaction towards the formation of the desired adduct $\mathbf{3 f}$ (entries 11 and 12) while limiting the formation of $\mathbf{3} \mathbf{f}^{\prime}$. Finally, functionalization of propylene carbonate $\mathbf{1 g}$, in which 50 equivalents of $\mathbf{1 g}$ were used regardless of the chosen photocatalyst, led to selective formation of $\mathbf{3 g}$ in $49 \%$ isolated yield in the presence of TBADT (entry 13), whereas the aromatic ketones failed to give any trace of products (entries 14 and 15).

The results reported above show some interesting trends. TBADT consistently demonstrated superior reactivity to that of the POCs used, because the desired adducts were obtained in the presence of a lower catalyst loading ( 2 $\mathrm{mol} \%$ versus $20 \mathrm{~mol} \%$ ) and a lower excess of the hydrogen donor (often 5-fold as against 50-fold). However, TBADT absorbs UV radiation exclusively, and it required the use of a solar-simulated light source, whereas the two POCs operated under irradiation by a violet light. Furthermore, thioxanthone was shown to be a superior photocatalyst to 9-fluorenone, as demonstrated by the higher conversions of the starting materials and the higher yields. All the photocatalysts showed a lower reactivity in the functionalization of 1b compared with that of $\mathbf{1 a}$ (with lower yields and/or a higher excess of hydrogen donor required). This might be due to steric effects connected with the presence of the two methyl groups at the acetalic position. Alternatively, it might be due to polar effects that disfavor the formation of the $\alpha$-oxyalkyl radical intermediate in $\mathbf{1 b}$ with respect to $\mathbf{1 a}$ due to the presence of the electron-withdrawing $\beta^{\prime}$-oxygen atom exerting a certain deactivating effect on HAT. ${ }^{24}$ Similarly, electronic effects played a role in the functionalization of cyclic carbonates (e.g., 1c), which required more-severe conditions (a greater excess of hydrogen donor) than in the case of $\mathbf{1 a}$ and $\mathbf{1 b}$, and reacted only in the presence of TBADT, the tested ketones being ineffective in this process. The low reactivity observed in the case of the ketones might be related to the $\pi \pi^{*}$ character of their lowest-lying 
triplet excited states, responsible for the key HAT step (as opposed to the $n \pi^{*}$ character of more-reactive ketones, such as benzophenone). ${ }^{25}$

Turning to the intramolecular selectivity aspect, we initially evaluated the competition between functionalization at the acetalic and the ethereal methylenic positions in 1d and $\mathbf{1 e}$, where an $\alpha, \alpha$-dioxyalkyl or an $\alpha$-oxyalkyl radical is involved, respectively. With these substrates, products $\mathbf{3}$ were consistently formed in higher amounts than products 4. The choice of the photocatalyst, however, had an effect, as TBADT gave $\mathbf{3}$ and $\mathbf{4}$ in a ratio of about 3 (statistically corrected), whereas for the ketones this value was $>18$ (statistically corrected). Indeed, a clean reaction occurred in the case of $\mathbf{1 e}$ in the presence of thioxanthone, where $\mathbf{3 e}$ was the only product formed. In the particular case of the functionalization of 1,3-dioxolane (1d) promoted by (aromatic) ketones, similar trends have been reported in the literature. Whereas some reports indicated a completely selective functionalization at the acetalic position, ${ }^{16,17 a-c}$ in a couple of cases, a distribution of products similar to that found here was described, notably in the acetone-initiated alkylation of 1,3-dioxolane by terminal olefins. ${ }^{26}$ Interestingly, the same selectivity pattern found here for aromatic ketones has also been observed in the functionalization of 1d through HAT promoted by a photogenerated chlorine atom. ${ }^{11 \mathrm{c}}$ On the other hand, the intramolecular competition between the functionalization at the acetalic versus the ethereal positions in the presence of TBADT has received limited attention. ${ }^{27}$ The different selectivity observed for the decatungstate anion compared with the tested POCs might be related to the different lifetimes of the relevant reactive states, where a short-lived species reacts with poor discrimination between the available sites. Indeed, whereas in the case of the decatungstate anion, the state responsible for the observed chemistry (named w0) has a lifetime of about $50-60$ ns in acetonitrile, ${ }^{28}$ the triplet states of the ketones employed here show much longer lifetimes, of the order of microseconds, ${ }^{25 a, c}$ in turn leading to predominant or exclusive functionalization at the acetalic position.

The presence of a methyl group, however, dramatically changed the reaction outcome, as observed in the case of $\mathbf{1 f}$. Thus, regardless of the choice of photocatalyst, the (tertiary) acetalic position was functionalized exclusively to give 3f in all cases. An undesired byproduct 3f' was observed, presumably formed through spontaneous deprotection of the dioxolane moiety of product $\mathbf{3 f}$. Its formation could, however, be suppressed by the addition of an (insoluble) inorganic base. Also, this agrees well with previous reports in which UV-light-induced, benzophenone-photosensitized, hydrogen abstraction from 2-alkyl-1,3-dioxolanes has been described as taking place at the 2-position exclusively. ${ }^{29}$ Finally, a similar selectivity towards the tertiary position was also observed in the case of propylene carbonate $(\mathbf{1 g})$, albeit only in the presence of TBADT. This is fully consistent with our previous investigation on lactones, where the presence of a tertiary position $\alpha$ to the oxygen atom drove the process toward exclusive functionalization of that position. ${ }^{30}$

In conclusion, the present work describes the different capabilities of selected HAT photocatalysts in promoting the functionalization of aliphatic oxygen heterocycles. We found that TBADT was the more reactive photocatalyst, requiring a lower loading and permitting the use of substrates in lower excesses than usually required for the aromatic ketones 9-fluorenone and thioxanthone. However, these ketones offer the possibility of performing more-selective reactions, as demonstrated in the reported examples of intramolecular selectivity.

\section{Funding Information}

We thank the MIUR for financial support (SIR Project 'Organic Synthesis via Visible Light Photocatalytic Hydrogen Transfer'; Code: RBSI145Y9R).

\section{Acknowledgment}

We thank Dr. Silvia Garbarino (University of Pavia) for preliminary experiments and Professor Stefano Protti (University of Pavia) for fruitful discussion.

\section{Supporting Information}

Supporting information for this article is available online at https://doi.org/10.1055/s-0037-1612079.

\section{References and Notes}

(1) Handbook of Heterocyclic Chemistry; Katritzky, A. R.; Ramsden, C. A.; Joule, J. A.; Zhdankin, V. V., Ed.; Elsevier: Oxford, 2010, 3rd ed..

(2) Delost, M. D.; Smith, D. T.; Anderson, B. J.; Njardarson, J. T. J. Med. Chem. 2018, 61, 10996.

(3) (a) Deiters, A.; Martin, S. F. Chem. Rev. 2004, 104, 2199. (b) Chemler, S. R.; Copeland, D. A. Top. Heterocycl. Chem. 2013, 32, 39. (c) Egi, M.; Akai, S. Heterocycles 2015, 91, 931. (d) Novák, Z.; Kotschy, A. Top. Heterocycl. Chem. 2014, 45, 231.

(4) Kaur, N. Synth. Commun. 2014, 44, 3483.

(5) Visible Light Photocatalysis in Organic Chemistry; Stephenson, C. R. J.; Yoon, T. P.; MacMillan, D. W. C., Ed.; Wiley-VCH: Weinheim, Germany, 2018.

(6) Ravelli, D.; Protti, S.; Fagnoni, M. Chem. Rev. 2016, 116, 9850.

(7) (a) Romero, N. A.; Nicewicz, D. A. Chem. Rev. 2016, 116, 10075. (b) Sideri, I. K.; Voutyritsa, E.; Kokotos, C. G. Org. Biomol. Chem. 2018, 16, 4596.

(8) Crespi, S.; Fagnoni, M. Top. Heterocycl. Chem. 2018, 54, 1.

(9) Special issue: Hydrogen Atom Transfer, Acc. Chem. Res., 2018, 51, 2601.

(10) (a) Capaldo, L.; Ravelli, D. Eur. J. Org. Chem. 2017, 2017, 2056. (b) Protti, S.; Fagnoni, M.; Ravelli, D. ChemCatChem 2015, 7, 1516. 
(11) For recent selected examples, see: (a) Deng, H.-P.; Zhou, Q.; Wu, J. Angew. Chem. Int. Ed. 2018, 57, 12661. (b) Le, C.; Liang, Y.; Evans, R. W.; Li, X.; MacMillan, D. W. C. Nature 2017, 547, 79. (c) Nielsen, M. K.; Shields, B. J.; Liu, J.; Williams, M. J.; Zacuto, M. J.; Doyle, A. G. Angew. Chem. Int. Ed. 2017, 56, 7191. (d) Shaw, M. H.; Shurtleff, V. W.; Terrett, J. A.; Cuthbertson, J. D.; MacMillan, D. W. C. Science 2016, 352, 1304. (e) Choi, G. J.; Zhu, Q.; Miller, D. C.; Gu, C. J.; Knowles, R. R. Nature 2016, 539, 268.

(12) Ravelli, D.; Protti, S.; Fagnoni, M. Acc. Chem. Res. 2016, 49, 2232.

(13) Ravelli, D.; Fagnoni, M.; Fukuyama, T.; Nishikawa, T.; Ryu, I. ACS Catal. 2018, 8, 701.

(14) Kamijo, S. Top. Heterocycl. Chem. 2018, 54, 71.

(15) Hoffmann, N. J. Phys. Org. Chem. 2015, 28, 121.

(16) Fan, X.-Z.; Rong, J.-W.; Wu, H.-L.; Zhou, Q.; Deng, H.-P.; Tan, J. D.; Xue, C.-W.; Wu, L.-Z.; Tao, H.-R.; Wu, J. Angew. Chem. Int. Ed. 2018, $57,8514$.

(17) (a) Manfrotto, C.; Mella, M.; Freccero, M.; Fagnoni, M.; Albini, A. J. Org. Chem. 1999, 64, 5024. (b) Geraghty, N. W. A.; Lally, A. Chem. Commun. 2006, 4300. (c) Dondi, D.; Protti, S.; Albini, A.; Mañas Carpio, S.; Fagnoni, M. Green Chem. 2009, 11, 1653. (d) Kamijo, S.; Kamijo, K.; Maruoka, K.; Murafuji, T. Org. Lett. 2016, 18, 6516. (e) Kawaai, K.; Yamaguchi, T.; Yamaguchi, E.; Endo, S.; Tada, N.; Ikari, A.; Itoh, A. J. Org. Chem. 2018, 83, 1988. (f) Papadopoulos, G. N.; Voutyritsa, E.; Kaplaneris, N.; Kokotos, C. G. Chem. Eur. J. 2018, 24, 1726.

(18) (a) Papadopoulos, G. N.; Limnios, D.; Kokotos, C. G. Chem. Eur. J. 2014, 20, 13811. (b) Papadopoulos, G. N.; Kokotos, C. G. J. Org. Chem. 2016, 81, 7023. (c) Papadopoulos, G. N.; Kokotos, C. G. Chem. Eur. J. 2016, 22, 6964.

(19) (a) Xia, J.-B.; Zhu, C.; Chen, C. J. Am. Chem. Soc. 2013, 135, 17494. (b) Xia, J.-B.; Zhu, C.; Chen, C. Chem. Commun. 2014, 50, 11701. (c) Bume, D. D.; Pitts, C. R.; Jokhai, R. T.; Lectka, T. Tetrahedron 2016, 72, 6031.

(20) Tachikawa, Y.; Cui, L.; Matsusaki, Y.; Tada, N.; Miura, T.; Itoh, A. Tetrahedron Lett. 2013, 54, 6218.

(21) C-H Functionalization of Aliphatic Oxygen Heterocycles; General Procedure

A solution of phenyl vinyl sulfone $(2 ; 0.1 \mathrm{M})$, the chosen aliphatic oxygen heterocycle $1(0.3-5 \mathrm{M})$, the photocatalyst (TBADT: $2 \mathrm{~mol} \%$; 9-fluorenone or thioxanthone: $20 \mathrm{~mol} \%$ ), and the base $\left(\mathrm{NaHCO}_{3}\right.$ or $\mathrm{Cs}_{2} \mathrm{CO}_{3} ; 1$ equiv, if required) in the chosen medium ( $\mathrm{MeCN}$ or $\mathrm{CH}_{2} \mathrm{Cl}_{2}$ ) was purged with $\mathrm{N}_{2}$ for 5 min then irradiated. The reaction course and the product distribution were monitored by GC analysis. The photolyzed solution was concentrated in vacuo, and the resulting residue was purified by column chromatography (silica gel, cyclohexane-EtOAc) to give product(s) 3 and/or 4.
4-[2-(Phenylsulfonyl)ethyl]-1,3-dioxolan-2-one (3c) Colorless oil; yield: $73 \mathrm{mg}$ (57\%). IR (neat): 2942, 1768, 1131 $\mathrm{cm}^{-1} .{ }^{1} \mathrm{H}$ NMR $\left(\mathrm{CDCl}_{3}, 300 \mathrm{MHz}\right): \delta=7.95-7.90(\mathrm{~m}, 2 \mathrm{H}), 7.75-$ $7.70(\mathrm{~m}, 1 \mathrm{H}), 7.65-7.60(\mathrm{~m}, 2 \mathrm{H}), 4.95-4.90(\mathrm{~m}, 1 \mathrm{H}), 4.65-4.50$ (m, $1 \mathrm{H}), 4.15-4.10(\mathrm{~m}, 1 \mathrm{H}), 3.35-3.20(\mathrm{~m}, 2 \mathrm{H}), 2.40-2.10(\mathrm{~m}, 2$ H). ${ }^{13} \mathrm{C} \mathrm{NMR}\left(\mathrm{CDCl}_{3}, 75 \mathrm{MHz}\right): \delta=154.4,138.4,134.2(\mathrm{CH}), 129.5$ $(\mathrm{CH}), 127.9(\mathrm{CH}), 74.5(\mathrm{CH}), 68.8\left(\mathrm{CH}_{2}\right), 51.6\left(\mathrm{CH}_{2}\right), 27.3\left(\mathrm{CH}_{2}\right)$. Anal. Calcd for $\mathrm{C}_{11} \mathrm{H}_{12} \mathrm{O}_{5} \mathrm{~S}$ : C, 51.55; H, 4.72. Found: C, 51.6; H, 4.7.

4-Methyl-4-[2-(phenylsulfonyl)ethyl]-1,3-dioxolan-2-one (3g) White solid; yield: $66 \mathrm{mg}$ (49\%); $\mathrm{mp} 117-118{ }^{\circ} \mathrm{C}$. IR (KBr): 2923 , $1801,1281,1064 \mathrm{~cm}^{-1} .{ }^{1} \mathrm{H}$ NMR $\left(\mathrm{CDCl}_{3}, 300 \mathrm{MHz}\right): \delta=7.95-$ 7.90 (m, $2 \mathrm{H}), 7.75-7.70(\mathrm{~m}, 1 \mathrm{H}), 7.65-7.60$ ( $\mathrm{m}, 2 \mathrm{H}), 4.25-4.15$ (m, $2 \mathrm{H}), 3.25-3.20(\mathrm{~m}, 2 \mathrm{H}), 2.25-2.20(\mathrm{~m}, 2 \mathrm{H}), 1.15(\mathrm{~s}, 3 \mathrm{H}) .{ }^{13} \mathrm{C}$ NMR $\left(\mathrm{CDCl}_{3}, 75 \mathrm{MHz}\right): \delta=153.5,138.4,134.2(\mathrm{CH}), 129.5(\mathrm{CH})$, 127.9 (CH), 81.5, $74.0(\mathrm{CH}), 50.7\left(\mathrm{CH}_{2}\right), 31.6\left(\mathrm{CH}_{2}\right), 24.2\left(\mathrm{CH}_{3}\right)$. Anal. Calcd for $\mathrm{C}_{12} \mathrm{H}_{14} \mathrm{O}_{5} \mathrm{~S}$ : C, 53.32; H, 5.22. Found: C, 53.3; $\mathrm{H}$, 5.2.

(22) Ravelli, D.; Montanaro, S.; Zema, M.; Fagnoni, M.; Albini, A. Adv. Synth. Catal. 2011, 353, 3295.

(23) The decatungstate anion is known to be unstable under strongly basic conditions, although it tolerates the presence of $\mathrm{NaHCO}_{3}$ as an insoluble base; see: Ravelli, D.; Albini, A.; Fagnoni, M. Chem. Eur. J. 2011, 17, 572

(24) Similar behavior has recently been observed in the case of HAT from alkanols and alkanediols; see: Salamone, M.; Ortega, V. B.; Martin, T.; Bietti, M. J. Org. Chem. 2018, 83, 5539.

(25) (a) Iyer, A.; Clay, A.; Jockusch, S.; Sivaguru, J. J. Phys. Org. Chem. 2017, 30, e3738. (b) Soep, B.; Mestdagh, J.-M.; Briant, M.; Gaveau, M.-A.; Poisson, L. Phys. Chem. Chem. Phys. 2016, 18, 22914. (c) Santhamurthy, A. R.; Rao, T. A. P.; Sobhanadri, J.; Murthy, V. R. K. J. Phys. Soc. Jpn. 1998, 67, 1220.

(26) (a) Rosenthal, I.; Elad, D. J. Org. Chem. 1968, 33, 805. (b) Graalfs, H.; Fröhlich, R.; Wolff, C.; Mattay, J. Eur. J. Org. Chem. 1999, 1057.

(27) Dondi, D.; Fagnoni, M.; Albini, A. Chem. Eur. J. 2006, 12, 4153.

(28) De Waele, V.; Poizat, O.; Fagnoni, M.; Bagno, A.; Ravelli, D. ACS Catal. 2016, 6, 7174.

(29) Mosca, R.; Fagnoni, M.; Mella, M.; Albini, A. Tetrahedron 2001 $57,10319$.

(30) Yamada, K.; Fukuyama, T.; Fujii, S.; Ravelli, D.; Fagnoni, M.; Ryu, I. Chem. Eur. J. 2017, 23, 8615. 
